\title{
Detection of acute myocardial infarct with T1-mapping post ferumoxytol contrast administration
}

\author{
Deneen Spatz ${ }^{1 *}$, Igor Klem¹, Lowie M Van Assche, Enn-Ling Chen ${ }^{1}$, Wolfgang G Rehwald², Han W Kim, \\ Christoph J Jensen ${ }^{1}$, David Wendell', Elizabeth Jenista', Raymond J Kim ${ }^{1}$
}

From 16th Annual SCMR Scientific Sessions

San Francisco, CA, USA. 31 January - 3 February 2013

\section{Background}

Gadolinium-enhanced CMR can be a useful adjunct in the diagnosis and assessment of acute myocardial infarction (AMI). However, gadolinium contrast is contraindicated in severe renal impairment. Alternative, non-gadolinium contrast agents for AMI assessment and contrast-enhanced CMR in general would be of substantial clinical importance. Ferumoxytol, an intravenous iron-supplementation drug, was approved in 2009 by the FDA for treatment of iron-deficiency anemia in patients with chronic kidney disease, and is known to have T1 shortening properties. The utility of T1-weighted ferumoxytol-enhanced CMR for detecting AMI has not been previously evaluated.

\section{Methods}

Six canines underwent acute LAD occlusion (70-minutes) with reperfusion. In-vivo CMR was performed four days post-MI on a 3.0T scanner in five animals (one animal died prior to acquisition of images). Clinically available Ferumoxytol $(30 \mathrm{mg} \mathrm{Fe} / \mathrm{ml})$ was administered to provide $5 \mathrm{mg} \mathrm{Fe} / \mathrm{kg}$ body weight. Short-axis T1-maps were acquired using a single-shot inversion-recovery, gradient-echo sequence with $>20$ inversion times ranging from 100-3000 ms. Acquisitions were separated by $>10$-second delay to allow full recovery of magnetization, and $\mathrm{T} 1$ times were determined by standard 3-parameter iterative curve-fitting. T1 maps were acquired serially at multiple time-points (5-8) following Ferumoxytolinjection (range 1-24 hours). Following imaging the hearts were removed for histopathologic analysis and the presence, location, and extent of AMI was validated by 2, 3, 5-triphenyltetrazolium chloride (TTC) staining.

${ }^{1}$ Duke University Cardiac MRI, Durham, NC, USA

Full list of author information is available at the end of the article

\section{Results}

All five animals had AMI verified by pathology, and mean infarct size was $12.7 \%$ (range 2.6-16.8\%) of LV mass. Ferumoxytol-kinetics of AMI was complex following two patterns (Figure 1, top). Pattern one $(n=3)$ demonstrated an early restriction of Ferumoxytol uptake in AMI regions (approx. 0-2 hrs post Ferumoxytol) compared with normal myocardium followed later by increased uptake in AMI relative to normal (approx. 2-6 hrs post), and finally washout of Ferumoxytol in both AMI and normal myocardium ( 24 hrs post). Pattern two $(n=2)$ demonstrated reduced Ferumoxytol uptake compared with normal myocardium

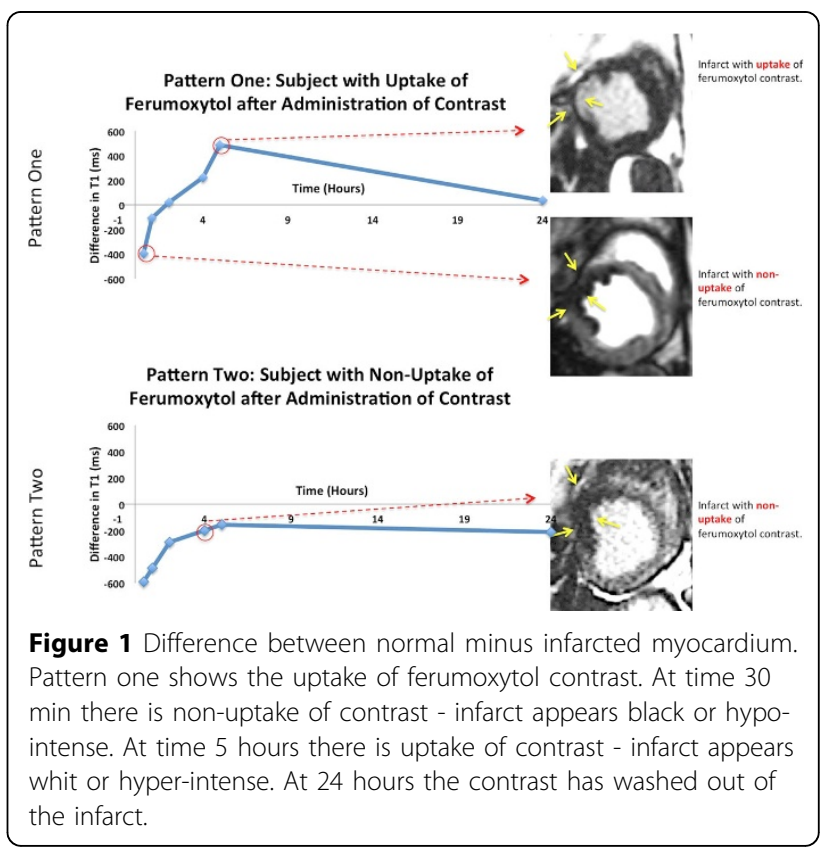

(c) 2013 Spatz et al; licensee BioMed Central Ltd. This is an Open Access article distributed under the terms of the Creative Commons 


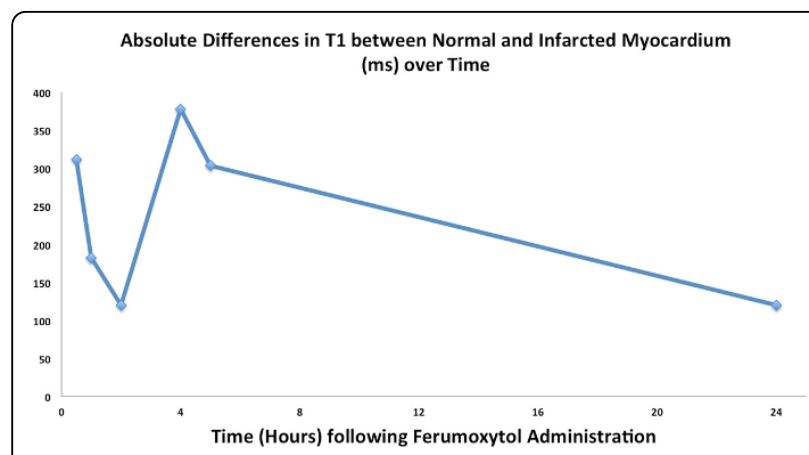

Figure 2 Absolute Difference in $\mathrm{T} 1$ between Normal and Infarcted Myocardium (ms) over Time after administration of Ferumoxytol contrast.

throughout all time points (Figure 1, bottom). The absolute difference in $\mathrm{T} 1$ between normal myocardium and AMI over time averaged for the five animals is shown in Figure 2. In 20 of 27 time-points (74\%), the absolute T1difference was $>100 \mathrm{~ms}$, which was sufficient to visualize AMI on routine inversion-recovery CMR.

\section{Conclusions}

Ferumoxytol kinetics in AMI are complex and may reflect either reduced or increased contrast uptake depending on time post contrast administration. However, AMI can consistently be detected by Ferumoxytol-enhanced CMR given positive absolute differences in $\mathrm{T} 1$.

\section{Funding}

5ROIHL064726-07.

\section{Author details}

'Duke University Cardiac MRI, Durham, NC, USA. ${ }^{2}$ Seimens, Chicago, IL, USA.

Published: 30 January 2013

doi:10.1186/1532-429X-15-S1-P173

Cite this article as: Spatz et al: Detection of acute myocardial infarct with T1-mapping post ferumoxytol contrast administration. Journal of Cardiovascular Magnetic Resonance 2013 15(Suppl 1):P173.
Submit your next manuscript to BioMed Central and take full advantage of:

- Convenient online submission

- Thorough peer review

- No space constraints or color figure charges

- Immediate publication on acceptance

- Inclusion in PubMed, CAS, Scopus and Google Scholar

- Research which is freely available for redistribution

Submit your manuscript at www.biomedcentral.com/submit 